\section{Crystal structure of (S)-2-amino-2- methylsuccinic acid}

\section{Isao Fujii}

School of Science, Tokai University, 4-1-1 Kitakaname, Hiratuka, Kanagawa 2591292, Japan. *Correspondence e-mail: fujii@wing.ncc.u-tokai.ac.jp

Received 3 September 2015; accepted 7 September 2015

Edited by H. Stoeckli-Evans, University of Neuchâtel, Switzerland

The title compound, $\mathrm{C}_{5} \mathrm{H}_{9} \mathrm{NO}_{4}$, crystallized as a zwitterion. There is an intramolecular $\mathrm{N}-\mathrm{H} \cdots \mathrm{O}$ hydrogen bond involving the trans-succinic acid and the ammonium group, forming an $S(6)$ ring motif. In the crystal, molecules are linked by $\mathrm{O}-\mathrm{H} \cdots \mathrm{O}$ hydrogen bonds, forming $C(7)$ chains along the $c$-axis direction. The chains are linked by $\mathrm{N}-\mathrm{H} \cdots \mathrm{O}$ and $\mathrm{C}-$ $\mathrm{H}$... O hydrogen bonds, forming sheets parallel to the $b c$ plane. Further $\mathrm{N}-\mathrm{H} \cdots \mathrm{O}$ hydrogen bonds link the sheets to form a three-dimensional framework.

Keywords: crystal structure; succinic acid; zwitterion; hydrogen bonding; three-dimensional framework.

CCDC reference: 1422827

\section{Related literature}

For general background and biological properties of 2-methylaspartic acid (MeASP), see: Pfeiffer \& Heinrich (1936); Delbaere et al. (1989); Nobe et al. (1998). For the absolute configuration and synthesis of the title compound, see: Terashima et al. (1966). For the crystal structure of related racemic compounds, see: Derricott et al. (1979); Brewer et al. (2013). For the crystal structure of DL-ASP, see: Flaig et al. (1998).

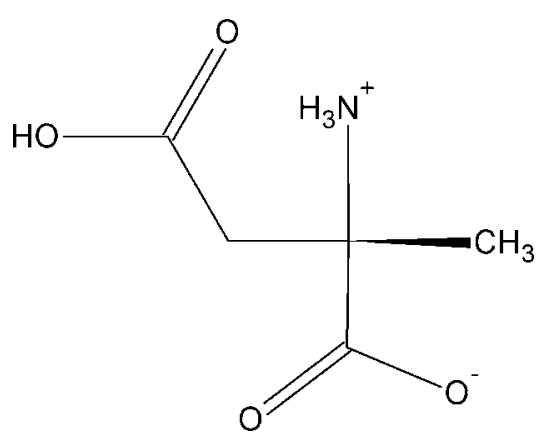

\section{Experimental}

2.1. Crystal data
$\mathrm{C}_{5} \mathrm{H}_{9} \mathrm{NO}_{4}$

$M_{r}=147.13$

Monoclinic, $C 2$

$a=8.3398$ (12) ^

$b=9.6725(10) \AA$

$c=8.0671(10) \AA$

$\beta=95.175(5)^{\circ}$

\subsection{Data collection}

Enraf-Nonius CAD-4 diffractometer

Absorption correction: $\psi$ scan (North et al., 1968)

843 measured reflections

\subsection{Refinement}

$R\left[F^{2}>2 \sigma\left(F^{2}\right)\right]=0.034$

$w R\left(F^{2}\right)=0.096$

$S=1.27$

700 reflections

109 parameters

2 restraints

$$
\begin{aligned}
& V=648.09(14) \AA^{3} \\
& Z=4 \\
& \mathrm{Cu} K \alpha \text { radiation } \\
& \mu=1.14 \mathrm{~mm}^{-1} \\
& T=297 \mathrm{~K} \\
& 0.4 \times 0.2 \times 0.2 \mathrm{~mm}
\end{aligned}
$$

$T_{\min }=0.76, T_{\max }=0.81$

Table 1

Hydrogen-bond geometry $\left(\AA{ }^{\circ}\right)$.

\begin{tabular}{lllll}
\hline$D-\mathrm{H} \cdots A$ & $D-\mathrm{H}$ & $\mathrm{H} \cdots A$ & $D \cdots A$ & $D-\mathrm{H} \cdots A$ \\
\hline $\mathrm{N} 1-\mathrm{H} 7 \cdots \mathrm{O} 4$ & $0.79(4)$ & $2.23(4)$ & $2.798(3)$ & $130(3)$ \\
$\mathrm{O} 3-\mathrm{H} 6 \cdots 1^{\mathrm{i}}$ & $0.84(4)$ & $1.70(4)$ & $2.543(2)$ & $177(5)$ \\
$\mathrm{N} 1-\mathrm{H} 7 \cdots \mathrm{O}^{\mathrm{ii}}$ & $0.79(4)$ & $2.53(4)$ & $3.093(3)$ & $130(3)$ \\
$\mathrm{N} 1-\mathrm{H} 8 \cdots \mathrm{O} 2^{\mathrm{iii}}$ & $0.86(3)$ & $1.90(4)$ & $2.754(3)$ & $170(3)$ \\
$\mathrm{N} 1-\mathrm{H} 9 \cdots 1^{\mathrm{i}}$ & $0.93(3)$ & $1.93(4)$ & $2.844(3)$ & $168(4)$ \\
$\mathrm{C} 3-\mathrm{H} 3 B \cdots \mathrm{O} 4^{\mathrm{v}}$ & 0.97 & 2.52 & $3.279(4)$ & 135 \\
\hline Symmetry codes: (i) $x, y, z+1 ;(\mathrm{ii})-x+\frac{1}{2}, y+\frac{1}{2},-z+1 ;\left(\right.$ iii) $-x+\frac{1}{2}, y+\frac{1}{2},-z ;$ (iv) \\
$-x, y,-z ;(\mathrm{v})-x+\frac{1}{2}, y-\frac{1}{2},-z+1$.
\end{tabular}

Data collection: $C A D-4$ Software (Enraf-Nonius, 1989); cell refinement: CAD-4 Software; data reduction: XCAD4 (Harms \& Wocadlo, 1995); program(s) used to solve structure: SHELXS97 (Sheldrick, 2008); program(s) used to refine structure: SHELXL2014 (Sheldrick, 2015); molecular graphics: ORTEP-3 for Windows (Farrugia, 2012) and Mercury (Macrae et al., 2008); software used to prepare material for publication: PLATON (Spek, 2003) and WinGX (Farrugia, 2012). 


\section{Acknowledgements}

The author thanks Tokai University for a research grant, which partially supported this work.

Supporting information for this paper is available from the IUCr electronic archives (Reference: SU5203).

\section{References}

Brewer, G., Burton, A. S., Dworkin, J. P. \& Butcher, R. J. (2013). Acta Cryst. E69, o1856-01857.

Delbaere, L. T., Kallen, J., Markovic-Housley, Z., Khomutov, A. R., Khomutov, R. M., Karpeisky, M. Y. \& Jansonius, J. N. (1989). Biochimie, 71, 449-459.
Derricott, C. \& Trotter, J. (1979). Acta Cryst. B35, 2230-2232.

Enraf-Nonius (1989). CAD-4 Software. Enraf-Nonius, Delft, The Netherlands.

Farrugia, L. J. (2012). J. Appl. Cryst. 45, 849-854.

Flaig, R., Koritsanszky, T., Zobel, D. \& Luger, P. (1998). J. Am. Chem. Soc. 120, 2227-2238.

Harms, K. \& Wocadlo, S. (1995). XCAD4. University of Marburg, Germany.

Macrae, C. F., Bruno, I. J., Chisholm, J. A., Edgington, P. R., McCabe, P., Pidcock, E., Rodriguez-Monge, L., Taylor, R., van de Streek, J. \& Wood, P. A. (2008). J. Appl. Cryst. 41, 466-470.

Nobe, Y., Kawaguchi, S., Ura, H., Nakai, T., Hirotsu, K., Kato, R. \& Kuramitsu, S. (1998). J. Biol. Chem. 273, 29554-29564.

North, A. C. T., Phillips, D. C. \& Mathews, F. S. (1968). Acta Cryst. A24, $351-$ 359.

Pfeiffer, P. \& Heinrich, E. (1936). J. Prakt. Chem. 146, 105-112.

Sheldrick, G. M. (2008). Acta Cryst. A64, 112-122.

Sheldrick, G. M. (2015). Acta Cryst. C71, 3-8.

Spek, A. L. (2003). J. Appl. Cryst. 36, 7-13.

Terashima, S., Achiwa, K. \& Yamada, S. (1966). Chem. Pharm. Bull. 14, 572 578. 


\section{supporting information}

Acta Cryst. (2015). E71, o731-o732［doi:10.1107/S2056989015016709]

\section{Crystal structure of (S)-2-amino-2-methylsuccinic acid}

\section{Isao Fujii}

\section{S1. Comment}

Solid-phase synthesis is now the accepted method to synthesis peptides, in which protected natural or non-natural amino acids are widely used; for example, 2-methylaspartic acid (MeASP) a non-natural amino acid. It has attracted attention as a substrate analog of aspartate aminotransferase (EC 2.6.1.1), and acts as a competitive inhibitor in the external aldimine (Delbaere et al., 1989; Nobe et al., 1998). Despite the biological and pharmaceutical interest, no crystal structures of MeASP derivatives have been reported except for the structure of DL-MeASP monohydrate (Brewer et al., 2013). In the title compound, Fig. 1, the succinic acid group has a trans-conformation $\left[\mathrm{C} 1-\mathrm{C} 2-\mathrm{C} 3-\mathrm{C} 4=-177.1(2)^{\circ}\right]$ versus. a cis-conformation [48.8 (4) ${ }^{\circ}$ ] in DL-MeASP. The carboxy group and the amino group make a hydrogen bonded half-chair $\mathrm{S}(6)$ ring motif (Table 1 and Fig. 1). The $\mathrm{S}(6)$ ring half-chair conformation and the trans-succinic acid arrangement are similar to the situation found in for DL-ASP (DLASPA03: Flaig et al. 1998).

In the crystal, molecules are linked by $\mathrm{O}-\mathrm{H} \cdots \mathrm{O}$ hydrogen bonds, involving the succinic acid groups, to form $\mathrm{C}(7)$ chains along the $c$ axis direction (Table 1 and Fig. 2). This is in contrast to the $\mathrm{N}-\mathrm{H} \cdots \mathrm{O}$ hydrogen bonded $\mathrm{C}(5)$ chains observed in the crystal structure of DL-MeASP. The chains are linked by $\mathrm{N}-\mathrm{H} \cdots \mathrm{O}$ and $\mathrm{C}-\mathrm{H} \cdots \mathrm{O}$ hydrogen bonds forming sheets parallel to the $b c$ plane. Further $\mathrm{N}-\mathrm{H} \cdots \mathrm{O}$ hydrogen bonds link the sheets to form a three-dimensional framework (Table 1 and Fig. 3). The methyl groups are surrounded by the hydrophilic planes and make a columnar structure (Fig. 3).

\section{S2. Synthesis and crystallization}

The title compound was purchased from Nagase-Sangyo Co. Ltd. The absolute configuration could not be established by anomalous-dispersion effects. The $(S)$ enantiomer has been chosen by referring the sign of known polarity in the synthetic procedure (Terashima et al., 1966). Rod-like colourless crystals of the title compound were obtained by vapour-phase diffusion of an ethanol-chloroform mixture at room temperature.

\section{S3. Refinement}

Crystal data, data collection and structure refinement details are summarized in Table 2. All the $\mathrm{H}$ atoms were located in difference Fourier maps. The $\mathrm{NH} 2$ and $\mathrm{OH} \mathrm{H}$ atoms were freely refined. The $\mathrm{C}$-bound $\mathrm{H}$ atoms were included in calculated positions and treated as riding atoms: $\mathrm{C}-\mathrm{H}=0.96-0.97 \AA$ with $U_{\text {iso }}(\mathrm{H})=1.5 \mathrm{U}_{\text {eq }}(\mathrm{C})$ for methyl $\mathrm{H}$ atoms and $1.2 \mathrm{U}_{\mathrm{eq}}(\mathrm{C})$ for other $\mathrm{H}$ atoms. 


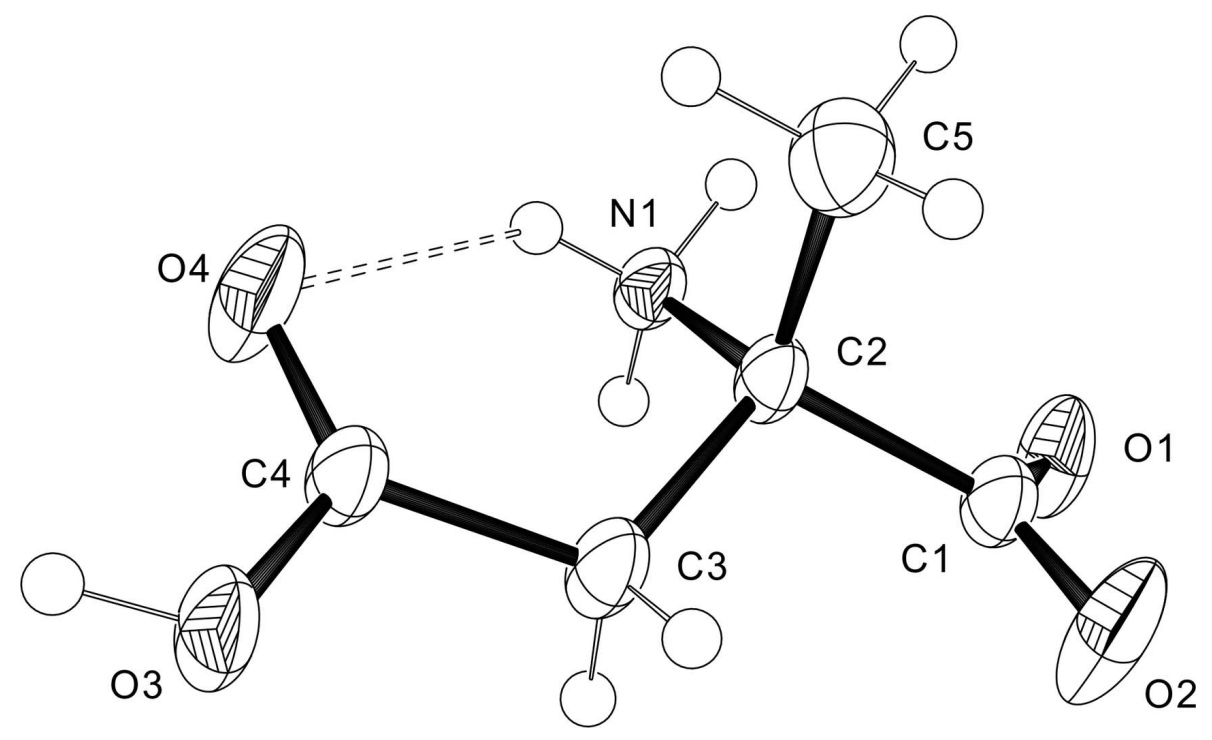

\section{Figure 1}

A view of the molecular structure of the title compound, with atom labelling. Displacement ellipsoids are drawn at the $50 \%$ probability level. The dashed line indicates the intramolecular $\mathrm{N}-\mathrm{H} \cdots \mathrm{O}$ hydrogen bond (see Table 1).

b

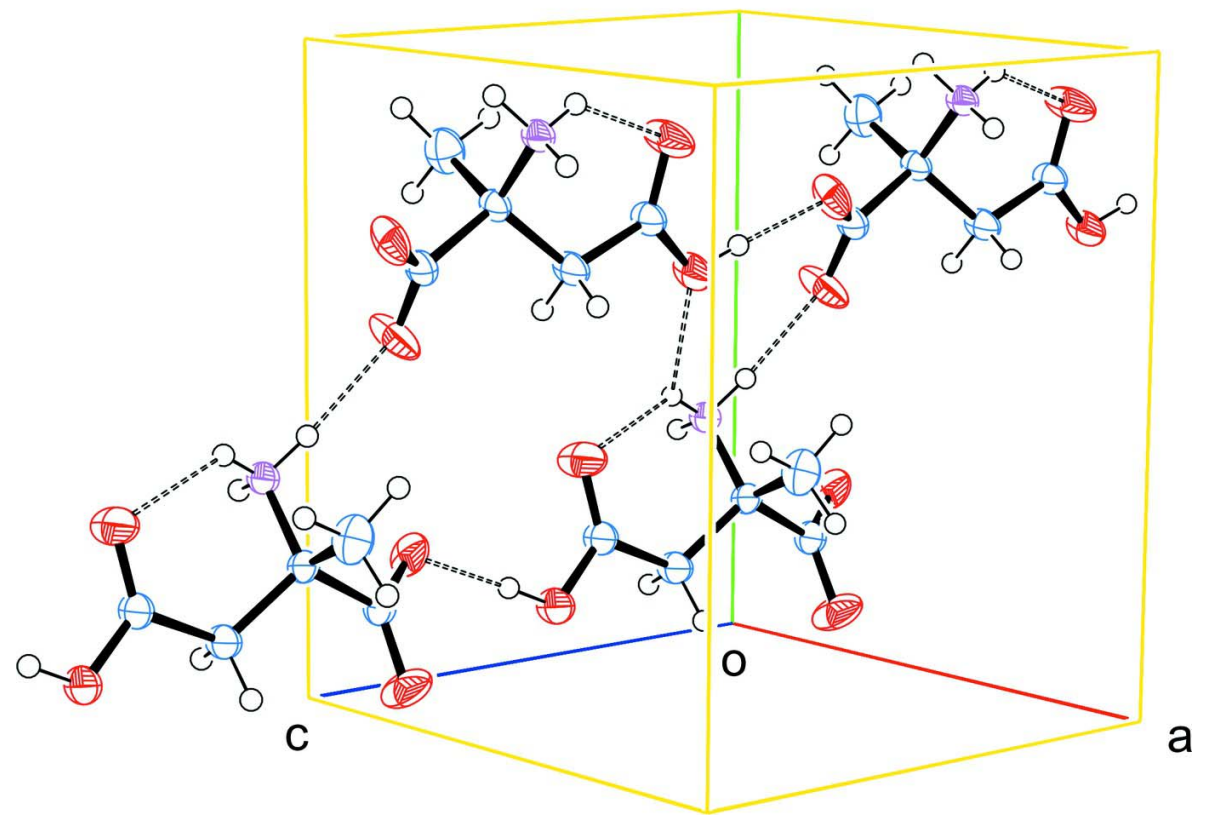

\section{Figure 2}

A partial view of the crystal packing of the title compound. Dashed lines indicate the $\mathrm{O}-\mathrm{H} \cdots \mathrm{O}$ and $\mathrm{N}-\mathrm{H} \cdots \mathrm{O}$ hydrogen bonds (see Table 1). 


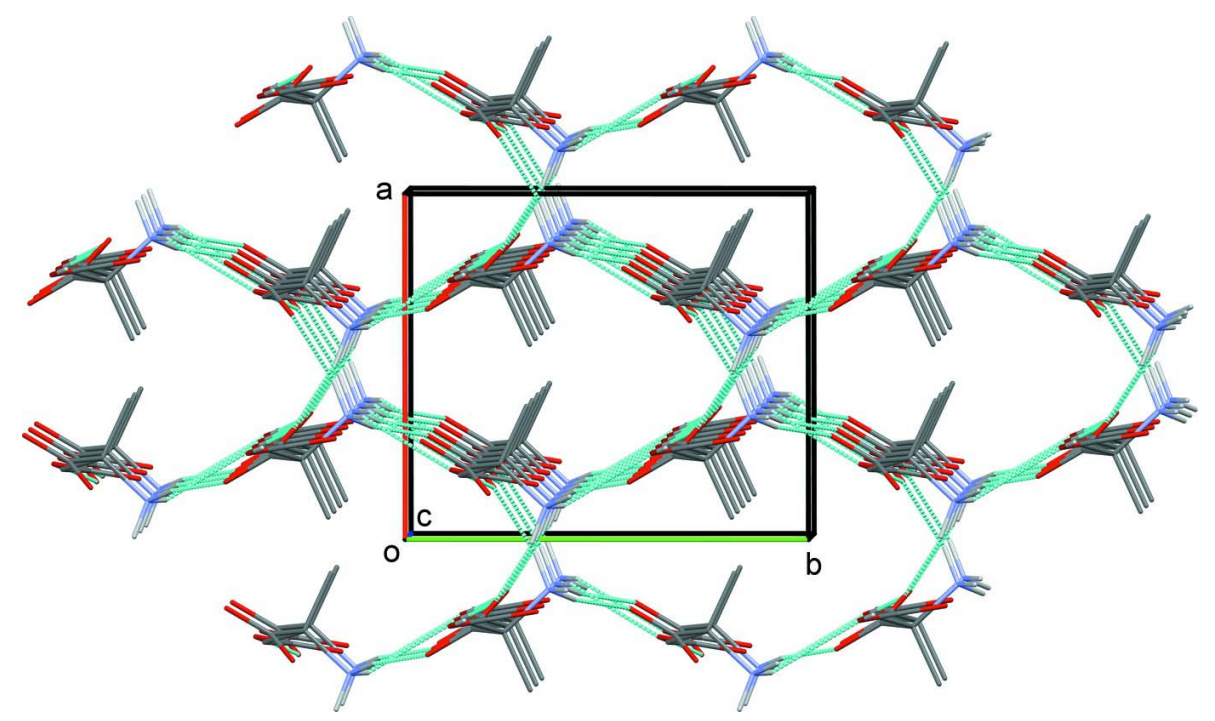

Figure 3

A view along the $c$ axis of the crystal packing of the title compound. Dashed lines indicate the $\mathrm{O}-\mathrm{H} \cdots \mathrm{O}$ and $\mathrm{N}-\mathrm{H} \cdots \mathrm{O}$ hydrogen bonds (see Table 1), and C-bound $\mathrm{H}$ atoms have been omitted for clarity.

\section{(S)-2-Amino-2-methylsuccinic acid}

Crystal data

$\mathrm{C}_{5} \mathrm{H}_{9} \mathrm{NO}_{4}$

$M_{r}=147.13$

Monoclinic, $C 2$

Hall symbol: C $2 \mathrm{y}$

$a=8.3398$ (12) $\AA$

$b=9.6725(10) \AA$

$c=8.0671(10) \AA$

$\beta=95.175(5)^{\circ}$

$V=648.09(14) \AA^{3}$

$Z=4$

\section{Data collection}

Enraf-Nonius CAD-4

diffractometer

Radiation source: sealed X-ray tube

Graphite monochromator

$\omega / 2 \theta$ scans

Absorption correction: $\psi$ scan

(North et al., 1968)

$T_{\min }=0.76, T_{\max }=0.81$

843 measured reflections

\section{Refinement}

Refinement on $F^{2}$

Least-squares matrix: full

$R\left[F^{2}>2 \sigma\left(F^{2}\right)\right]=0.034$

$w R\left(F^{2}\right)=0.096$

$S=1.27$

700 reflections
$F(000)=312$

$D_{\mathrm{x}}=1.508 \mathrm{Mg} \mathrm{m}^{-3}$

$\mathrm{Cu} K \alpha$ radiation, $\lambda=1.54178 \AA$

Cell parameters from 25 reflections

$\theta=20-28^{\circ}$

$\mu=1.14 \mathrm{~mm}^{-1}$

$T=297 \mathrm{~K}$

Rod, colorless

$0.4 \times 0.2 \times 0.2 \mathrm{~mm}$

700 independent reflections

699 reflections with $I>2 \sigma(I)$

$R_{\text {int }}=0.019$

$\theta_{\max }=74.0^{\circ}, \theta_{\min }=5.5^{\circ}$

$h=-10 \rightarrow 1$

$k=-12 \rightarrow 0$

$l=-10 \rightarrow 10$

3 standard reflections every 300 reflections intensity decay: none

109 parameters

2 restraints

Primary atom site location: structure-invariant direct methods

Secondary atom site location: difference Fourier map 
Hydrogen site location: mixed

$\mathrm{H}$ atoms treated by a mixture of independent and constrained refinement

$w=1 /\left[\sigma^{2}\left(F_{\mathrm{o}}^{2}\right)+(0.0559 P)^{2}+0.2563 P\right]$

where $P=\left(F_{\mathrm{o}}^{2}+2 F_{\mathrm{c}}^{2}\right) / 3$

$(\Delta / \sigma)_{\max }<0.001$
$\Delta \rho_{\max }=0.29{\mathrm{e} \AA^{-3}}^{-3}$

$\Delta \rho_{\min }=-0.21$ e $\AA^{-3}$

Extinction correction: SHELXL2014 (Sheldrick, 2014), $\mathrm{Fc}^{*}=\mathrm{kFc}\left[1+0.001 \times \mathrm{xc}^{2} \lambda^{3} / \sin (2 \theta)\right]^{-1 / 4}$

Extinction coefficient: 0.045 (4)

\section{Special details}

Geometry. All e.s.d.'s (except the e.s.d. in the dihedral angle between two 1.s. planes) are estimated using the full covariance matrix. The cell e.s.d.'s are taken into account individually in the estimation of e.s.d.'s in distances, angles and torsion angles; correlations between e.s.d.'s in cell parameters are only used when they are defined by crystal symmetry. An approximate (isotropic) treatment of cell e.s.d.'s is used for estimating e.s.d.'s involving 1.s. planes.

Fractional atomic coordinates and isotropic or equivalent isotropic displacement parameters $\left(\AA^{2}\right)$

\begin{tabular}{|c|c|c|c|c|}
\hline & $x$ & $y$ & $z$ & $U_{\text {iso }} * / U_{\text {eq }}$ \\
\hline H8 & $0.155(3)$ & $0.434(4)$ & $0.122(4)$ & $0.026(7)^{*}$ \\
\hline $\mathrm{H} 7$ & $0.126(4)$ & $0.416(4)$ & $0.275(5)$ & $0.040(9)^{*}$ \\
\hline H6 & $0.207(5)$ & $0.170(5)$ & $0.725(4)$ & $0.071(14)^{*}$ \\
\hline H9 & $0.022(4)$ & $0.340(4)$ & $0.164(4)$ & $0.040(9)^{*}$ \\
\hline $\mathrm{C} 1$ & 0.2417 & $0.1802(3)$ & 0.0388 & $0.0273(5)$ \\
\hline $\mathrm{C} 2$ & 0.2485 & $0.2608(2)$ & 0.2046 & $0.0238(5)$ \\
\hline $\mathrm{C} 3$ & $0.2132(3)$ & $0.1603(3)$ & 0.3436 & $0.0305(6)$ \\
\hline $\mathrm{H} 3 \mathrm{~A}$ & 0.1108 & 0.1156 & 0.3124 & $0.037 *$ \\
\hline H3B & 0.2954 & 0.0891 & 0.3512 & $0.037^{*}$ \\
\hline $\mathrm{C} 4$ & $0.2069(3)$ & $0.2244(3)$ & $0.5137(3)$ & $0.0284(6)$ \\
\hline $\mathrm{C} 5$ & $0.4148(3)$ & $0.3267(4)$ & $0.2343(4)$ & $0.0386(7)$ \\
\hline $\mathrm{H} 5 \mathrm{~A}$ & 0.4277 & 0.3947 & 0.1498 & $0.058 *$ \\
\hline H5B & 0.4959 & 0.2567 & 0.2301 & $0.058^{*}$ \\
\hline $\mathrm{H} 5 \mathrm{C}$ & 0.4254 & 0.3702 & 0.3417 & $0.058^{*}$ \\
\hline N1 & 0.1250 & $0.3741(2)$ & $0.1916(3)$ & $0.0248(5)$ \\
\hline $\mathrm{O} 1$ & $0.1677(2)$ & $0.2358(2)$ & $-0.0871(2)$ & $0.0378(5)$ \\
\hline $\mathrm{O} 2$ & $0.3164(3)$ & $0.0705(2)$ & $0.0427(3)$ & $0.0525(7)$ \\
\hline $\mathrm{O} 3$ & $0.2242(3)$ & 0.1339 (2) & $0.6334(2)$ & $0.0392(6)$ \\
\hline $\mathrm{O} 4$ & $0.1831(4)$ & $0.3463(2)$ & $0.5370(2)$ & $0.0554(7)$ \\
\hline
\end{tabular}

Atomic displacement parameters $\left(\AA^{2}\right)$

\begin{tabular}{lllllll}
\hline & $U^{11}$ & $U^{22}$ & $U^{33}$ & $U^{12}$ & $U^{13}$ & $U^{23}$ \\
\hline $\mathrm{C} 1$ & $0.0384(11)$ & $0.0263(12)$ & $0.0177(10)$ & $0.0010(10)$ & $0.0051(8)$ & $-0.0020(9)$ \\
$\mathrm{C} 2$ & $0.0349(10)$ & $0.0220(11)$ & $0.0147(10)$ & $0.0029(9)$ & $0.0028(8)$ & $-0.0013(8)$ \\
$\mathrm{C} 3$ & $0.0520(14)$ & $0.0239(13)$ & $0.0157(10)$ & $0.0039(11)$ & $0.0039(9)$ & $-0.0005(9)$ \\
$\mathrm{C} 4$ & $0.0436(13)$ & $0.0252(12)$ & $0.0164(10)$ & $0.0015(10)$ & $0.0025(9)$ & $-0.0014(9)$ \\
$\mathrm{C} 5$ & $0.0342(12)$ & $0.0476(17)$ & $0.0338(13)$ & $-0.0022(12)$ & $0.0020(10)$ & $-0.0065(12)$ \\
$\mathrm{N} 1$ & $0.0368(11)$ & $0.0213(10)$ & $0.0164(9)$ & $0.0008(8)$ & $0.0036(7)$ & $-0.0007(8)$ \\
O1 & $0.0510(10)$ & $0.0455(11)$ & $0.0167(8)$ & $0.0152(9)$ & $0.0011(7)$ & $-0.0039(8)$ \\
O2 & $0.0930(17)$ & $0.0383(13)$ & $0.0257(10)$ & $0.0290(13)$ & $0.0023(10)$ & $-0.0082(9)$ \\
O3 & $0.0721(13)$ & $0.0304(10)$ & $0.0162(9)$ & $0.0085(9)$ & $0.0096(8)$ & $0.0010(8)$ \\
O4 & $0.118(2)$ & $0.0290(12)$ & $0.0200(9)$ & $0.0126(12)$ & $0.0102(10)$ & $-0.0024(8)$ \\
& & & & & &
\end{tabular}


Geometric parameters $(\AA, \stackrel{\circ}{)})$

\begin{tabular}{llll}
\hline $\mathrm{C} 1-\mathrm{O} 2$ & $1.229(3)$ & $\mathrm{C} 4-\mathrm{O} 4$ & $1.213(4)$ \\
$\mathrm{C} 1-\mathrm{O} 1$ & $1.261(3)$ & $\mathrm{C} 4-\mathrm{O} 3$ & $1.301(3)$ \\
$\mathrm{C} 1-\mathrm{C} 2$ & $1.545(3)$ & $\mathrm{C} 5-\mathrm{H} 5 \mathrm{~A}$ & 0.9600 \\
$\mathrm{C} 2-\mathrm{N} 1$ & $1.502(3)$ & $\mathrm{C} 5-\mathrm{H} 5 \mathrm{~B}$ & 0.9600 \\
$\mathrm{C} 2-\mathrm{C} 5$ & $1.525(3)$ & $\mathrm{C} 5-\mathrm{H} 5 \mathrm{C}$ & 0.9600 \\
$\mathrm{C} 2-\mathrm{C} 3$ & $1.532(3)$ & $\mathrm{N} 1-\mathrm{H} 8$ & $0.86(4)$ \\
$\mathrm{C} 3-\mathrm{C} 4$ & $1.511(3)$ & $\mathrm{N} 1-\mathrm{H} 7$ & $0.78(4)$ \\
$\mathrm{C} 3-\mathrm{H} 3 \mathrm{~A}$ & 0.9700 & $\mathrm{~N} 1-\mathrm{H} 9$ & $0.93(4)$ \\
$\mathrm{C} 3-\mathrm{H} 3 \mathrm{~B}$ & 0.9700 & $\mathrm{O} 3-\mathrm{H} 6$ & $0.84(2)$ \\
& & & \\
$\mathrm{O} 2-\mathrm{C} 1-\mathrm{O} 1$ & $126.8(2)$ & $\mathrm{O} 4-\mathrm{C} 4-\mathrm{C} 3$ & $124.0(2)$ \\
$\mathrm{O} 2-\mathrm{C} 1-\mathrm{C} 2$ & $115.7(2)$ & $\mathrm{O} 3-\mathrm{C} 4-\mathrm{C} 3$ & $112.8(2)$ \\
$\mathrm{O} 1-\mathrm{C} 1-\mathrm{C} 2$ & $117.3(2)$ & $\mathrm{C} 2-\mathrm{C} 5-\mathrm{C} 5-\mathrm{H} 5 \mathrm{~B}$ & 109.5 \\
$\mathrm{~N} 1-\mathrm{C} 2-\mathrm{C} 5$ & $108.3(2)$ & $\mathrm{H} 5 \mathrm{~A}-\mathrm{C} 5-\mathrm{H} 5 \mathrm{~B}$ & 109.5 \\
$\mathrm{~N} 1-\mathrm{C} 2-\mathrm{C} 3$ & $109.79(18)$ & $\mathrm{C} 2-\mathrm{C} 5-\mathrm{H} 5 \mathrm{C}$ & 109.5 \\
$\mathrm{C} 5-\mathrm{C} 2-\mathrm{C} 3$ & $112.5(2)$ & $\mathrm{H} 5 \mathrm{~A}-\mathrm{C} 5-\mathrm{H} 5 \mathrm{C}$ & 109.5 \\
$\mathrm{~N} 1-\mathrm{C} 2-\mathrm{C} 1$ & $109.67(18)$ & $\mathrm{H} 5 \mathrm{~B}-\mathrm{C} 5-\mathrm{H} 5 \mathrm{C}$ & 109.5 \\
$\mathrm{C} 5-\mathrm{C} 2-\mathrm{C} 1$ & $107.98(18)$ & $\mathrm{C} 2-\mathrm{N} 1-\mathrm{H} 8$ & 109.5 \\
$\mathrm{C} 3-\mathrm{C} 2-\mathrm{C} 1$ & $108.60(19)$ & $\mathrm{H} 8-\mathrm{N} 1-\mathrm{H} 7$ & $107(2)$ \\
$\mathrm{C} 4-\mathrm{C} 3-\mathrm{C} 2$ & $115.4(2)$ & $\mathrm{C} 2-\mathrm{N} 1-\mathrm{H} 9$ & $112(3)$ \\
$\mathrm{C} 4-\mathrm{C} 3-\mathrm{H} 3 \mathrm{~A}$ & 108.4 & $\mathrm{H} 8-\mathrm{N} 1-\mathrm{H} 9$ & $104(3)$ \\
$\mathrm{C} 2-\mathrm{C} 3-\mathrm{H} 3 \mathrm{~A}$ & 108.4 & $\mathrm{H} 7-\mathrm{N} 1-\mathrm{H} 9$ & $112(3)$ \\
$\mathrm{C} 4-\mathrm{C} 3-\mathrm{H} 3 \mathrm{~B}$ & 108.4 & & $113(3)$ \\
$\mathrm{C} 2-\mathrm{C} 3-\mathrm{H} 3 \mathrm{~B}$ & 108.4 & $109(3)$ \\
$\mathrm{H} 3 \mathrm{~A}-\mathrm{C} 3-\mathrm{H} 3 \mathrm{~B}$ & 107.5 & $111(4)$ \\
$\mathrm{O} 4-\mathrm{C} 4-\mathrm{O} 3$ & $123.2(2)$ & & \\
& & &
\end{tabular}

Hydrogen-bond geometry $\left(\AA,{ }^{\circ}\right)$

\begin{tabular}{lllll}
\hline$D-\mathrm{H} \cdots A$ & $D-\mathrm{H}$ & $\mathrm{H} \cdots A$ & $D \cdots A$ & $D-\mathrm{H} \cdots A$ \\
\hline $\mathrm{N} 1-\mathrm{H} 7 \cdots \mathrm{O} 4$ & $0.79(4)$ & $2.23(4)$ & $2.798(3)$ & $130(3)$ \\
$\mathrm{O} 3-\mathrm{H} 6 \cdots \mathrm{O} 1^{\mathrm{i}}$ & $0.84(4)$ & $1.70(4)$ & $2.543(2)$ & $177(5)$ \\
$\mathrm{N} 1-\mathrm{H} 7 \cdots 3^{\mathrm{ii}}$ & $0.79(4)$ & $2.53(4)$ & $3.093(3)$ & $130(3)$ \\
$\mathrm{N} 1-\mathrm{H} 8 \cdots \mathrm{O} 2^{\mathrm{iii}}$ & $0.86(3)$ & $1.90(4)$ & $2.754(3)$ & $170(3)$ \\
$\mathrm{N} 1-\mathrm{H} 9 \cdots{ }^{\mathrm{iv}}$ & $0.93(3)$ & $1.93(4)$ & $2.844(3)$ & $168(4)$ \\
$\mathrm{C} 3-\mathrm{H} 3 B \cdots \mathrm{O} 4^{\mathrm{v}}$ & 0.97 & 2.52 & $3.279(4)$ & 135
\end{tabular}

Symmetry codes: (i) $x, y, z+1$; (ii) $-x+1 / 2, y+1 / 2,-z+1$; (iii) $-x+1 / 2, y+1 / 2,-z$; (iv) $-x, y,-z$; (v) $-x+1 / 2, y-1 / 2,-z+1$. 